\title{
Genetic risk factor identified for cystic fibrosis liver disease
}

Patients with cystic fibrosis who carry the SERPINA1 $\mathrm{Z}$ allele have an approximate fivefold increased risk for developing severe liver disease and portal hypertension, according to a new study.

Genes unrelated to the cystic fibrosis gene, CFTR, may contribute to the risk for cystic fibrosis liver disease (CFLD). Jaclyn Bartlett and colleagues conducted an international, multicenter study to determine associations between 9 polymorphisms in 5 non-CFTR genes and CFLD. Initial analysis of 124 CFLD patients and 834 controls (patients with CF but without liver disease aged $\geq 15$ years), and a replication study with 136 CFLD patients and 1,088 controls revealed CFLD to be associated with the SERPINA $1 Z$ allele. "A striking increased risk [odds ratio 5.04] for liver disease that was so severe it frequently warranted liver transplantation was found in patients carrying the SERPINA1 Z allele," says Bartlett, lead investigator.

The authors are working to define the mechanism whereby the $\mathrm{Z}$ allele causes severe liver disease as it might suggest novel treatments. The group is also studying other genetic variants that predispose to the development of CFLD. "Discovery of genetic risk factors for CFLD offers the possibility of identifying infants with cystic fibrosis who are at risk for liver disease, and provides the opportunity to develop preventative therapies," explains Bartlett.

\section{Rachel Jones}

Original article Bartlett, J. R. et al. Genetic modifiers of liver disease in cystic fibrosis. JAMA 302, 1076-1083 (2009). 